since January/2007 to September/2012, intersecting the terms 'medicinal gases' and 'medical gases'.

Results A total of 6 medicinal gases currently available in Portugal were analysed: medicinal air, nitric oxide, nitrous oxide, nitrous oxide/oxygen, oxygen and xenon. The main interactions of these gases with other medicinal products are: i) nitric oxide: oxygen, almitrine, nitroglycerin, sodium nitroprusside, phenylephrine, phosphodiesterase inhibitors, prilocaine, sulfonamides; ii) nitrous oxide: cyanocobalamin, drugs that depress the central nervous system (CNS), methotrexate; iii) oxygen: antiarrhythmics, bleomycin, chloroquine, chlorpromazine, corticosteroids, dactinomycin, doxorubicin, nitrofurantoin, phytomenadione, sympathomimetics; iv) xenon: antihypertensives, drugs that depress the CNS, other inhaled anaesthetic agents, sympathomimetics. No interactions were found with medicinal air. The database developed also describes the interaction mechanisms for each medicinal gas with each drug mentioned and the measures recommended to prevent major side effects.

Conclusions The database produced is a valuable tool for Portuguese hospital pharmacists who dispense medicinal gases, contributing to validating prescriptions for these medicines quickly and effectively.

No conflict of interest.

\section{GRP-105 INTRODUCTION OF A MEDICINES RECONCILIATION PROGRAMMIE IN THE ORTHOPAEDIC SURGERY UNIT}

doi:10.1136/ejhpharm-2013-000276.105

'L Soriano, ${ }^{1} F J$ Rodríguez-Lucena, ' $\mathrm{C}$ Matoses Chirivella, ${ }^{1} \mathrm{~A}$ Murcia López, ${ }^{2} \mathrm{PJ}$ MoyaForcén, 'I Triano Garcia, 'R Gutierrez Vozmediano, 'A Martínez Valero, 'R Antón Torres, 'A Navarro Ruíz. 'Hospital General Universitario de Elche, Pharmacy Service, Elche, Spain; ${ }^{2}$ Hospital General Universitario de Elche, Department of surgery, Elche, Spain

Background The average hospitalised patient is subject to at least one medicines error per day. More than $40 \%$ of medicines errors are believed to result from inadequate medicines reconciliation.

Purpose To investigate the introduction of a medicines reconciliation programme in the orthopaedic surgery unit.

Materials and Methods January 2010-March 2012. The patient selection criteria were $\geq 65$ years old, home treatments $\geq 5$ drugs and anticipated hospital stay $\geq 3$ days. The reconciliation treatment was also performed for any other patients when requested by the doctor. Patients were found to be sensitive to the reconciliation by the pharmacist. Any Drug Related Problems (DRPs) detected were recorded and categorised. A prescription was given with the home treatment, with the aim of continuing treatment, discontinuing it or performing a therapeutic exchange. The process ended with oral and written pharmacotherapeutic information on the day of discharge.

Results Medicines reconciliation was carried out on 300 patients with an average age of 75.86 , average stay of 9.57 days and distribution by gender 224 women (75\%) and 76 men (25\%). The number of medicines/patient was 6.57 . During the prescription by the pharmacist, 1058 drugs were provided according to guidelines, 276 were suspended and in 663 cases a therapeutic exchange was performed. As regards the DRPs detected, 50 were caused on admittance and 15 at discharge. The DRPs were classified as follows: safety 51, effectiveness 10, adherence 2 and indication 2. Types of DRP: overdose 17 , adverse reaction 4 , need of extra treatment 6 , unnecessary medicine 23, unsuitable drug 10, insufficient dosing 4, not dispensed 1. As to the seriousness of the DRPs: class 1: 5 patients didn't use the medicines that they needed; class 2, 24 patients used medicines that they didn't need; class 3, 23 patients used an erroneously chosen medicine; class 4,10 patients used an erroneously chosen medicine; class 5,3 patients used a lower dose and/or a different dosage schedule from that required and/or don't continue treatment for the full duration of the treatment indicated, according to the Granada consensus of 1998.
Conclusions Participation of the pharmacist in the reconciliation of treatment allows DRPs to be detected at admission and discharge and educated the patient on his or her treatment at discharge from the hospital.

No conflict of interest.

\section{GRP-106 INVOLVEMENT OF THE PHARMACY AND THERAPEUTICS COMMITTEE IN CLINICAL DECISION SUPPORT SYSTEMS FOCUSED ON ANTICOAGULANTS}

doi:10.1136/ejhpharm-2013-000276.106

M Touleimat, D Cirotteau, P Lorillon, N Borgnis-Desbordes. Brest University Hospital, Pharmacy, Brest, France

Background Adverse drug events related to anticoagulants are common and clinically significant. Computerized physician order entry (CPOE) and clinical decision support systems (CDSSs) are widely viewed as crucial for reducing prescribing errors.

Purpose To make prescriptions safer and to promote good practise, by developing CDSSs focused on oral and injectable anticoagulants. Materials and Methods A review was carried out of existing guidelines and practise in the units.

About ten meetings with clinicians (cardiologists, thrombosis specialists) and pharmacists from the Pharmacy and Therapeutics Committee (PTC) were required to write these CDSSs.

The CDSSs were presented and tested in the cardiology units. New discussions and improvements in the CDSSs were made with prescribers, nurses and pharmacists.

The final CDSSs were validated by the Pharmacy and Therapeutics Committee (PTC).

Results Nine CDSSs had already been validated by the PTC: Vitamin K Antagonist (VKA), heparin sodium, heparin calcium, Low Molecular Weight Heparins (LMWHs) in prophylactic and curative treatment of deep-vein thrombosis and pulmonary embolism, LMWHs for acute coronary syndrome ST-segment elevation myocardial infarction and non-ST-segment elevation myocardial infarction, LMWHs for cardiac arrhythmia, and treatment of heparin-induced thrombocytopenia.

There are still regular meetings to develop CDSSs on new anticoagulants: dabigatran, rivaroxaban and apixaban.

Each CDSS provides:

- Information on the choice of a therapeutic strategy based on the indication and the clinical context.

- Usual doses and rates of administration.

- A dose calculation based on weight (heparins).

- Overrun alerts when the dose is exceeded.

- Regular laboratory tests at the recommended frequency.

- Protocols for dosage adjustments based on the biological values.

- Administration modalities for the nurses.

Since the implementation of the CDSS on VKA, annual fluindione prescriptions have decreased by $17 \%$ and annual warfarin prescriptions have increased by $53 \%$ in accordance with the recommendation to prescribe warfarin as the first-line oral anticoagulant.

Conclusions Development of CDSSs referred to by the CPOE system takes a lot of time but is a good way of disseminating PTC guidelines to all prescribers, pharmacists and nurses. CDSSs can assist clinicians in the management of patients requiring anticoagulant treatment by improving compliance with care standards. These CDSSs are updated following changes in guidelines and clinical practise. Other CDSSs focused on high-alert medicines will be introduced when computerised prescribing is implemented for the entire hospital.

No conflict of interest. 